\title{
Comentario sobre investigación en sistemas de salud, atención primaria de salud y participación para la transformación social
}

\author{
Comment on investigation of health systems, health primary care, and participation to \\ social change
}

Françoise Barten ${ }^{1}$

${ }^{1}$ Doctor of Medicine; Senior researcher at Radboud University Nijmegen Medical Centre, Department of Primary and Community Care, International Public Health, Nijmegen International Center for Health Systems Research and Education (NICHE), Nijmegen Urban Health Group - Nijmegen, the Netherlands.

francoise189@gmail.com
Los Sistemas de Salud han experimentado diferentes cambios recientemente y pocas veces estos han sido basados en evidencia. Factores a nivel global cada vez más inciden en la política nacional de salud, mientras que el escenario se complejiza con la llegada de nuevos y poderosos actores (STUCKLER et al., 2011). Una mejor comprensión de funciones, elementos, actores, proceso, estructura y fuerzas motrices es imprescindible para incrementar la relevancia, la efectividad y la eficiencia de los sistemas y para mejorar la salud. La investigación de sistemas y de políticas de salud adquiere por lo tanto una relevancia particular para reducir la brecha entre discurso y practica y así poder incidir en la determinación de las inequidades en salud.

Al ser un campo relativamente nuevo, la investigación de sistemas de salud también enfrenta muchos retos. Ejemplos interesantes fueron los estudios "Rescate" o "Desarrollo de Sistemas de Salud en Centro América, con énfasis en los esfuerzos desarrollados desde la sociedad civil" (2001) - desarrollado posterior a la firma de los acuerdos de paz en Guatemala, un poco en contra de la corriente de aquella época de reforma del sector salud. El objetivo fue estudiar el desarrollo de los sistemas de salud desde la perspectiva de la sociedad civil, basados en los principios de la atención primaria de salud (APS), Salud para Todos. La investigación con universidades en Nicaragua y El Salvador, involucrando a organizaciones de la sociedad civil y de tomadores de decisión en estos países y en Guatemala, inmediatamente después del conflicto armado, se convirtió en un proceso de aprendizaje colectivo importante.

En El Salvador, la decisión de priorizar el estudio a profundidad en las experiencias de Guargila y de la Zona Norte en San Salvador fue producto de un proceso participativo que involucro a otros actores, después de haber mapeado a más de 100 experiencias en el país. En ese mismo período, el sistema nacional de Colombia introducía cambios importantes en la organización del aseguramiento y en la prestación de servicios de salud de la población. Rodríguez y Garzón (2009) señalaron que el enfoque de "atención a la comunidad y al entorno" se sustituyó por la atención al "afiliado", mientras que las necesidades básicas en salud se convirtieron en "el mercado de la salud" y los presupuestos para las necesidades de salud quedaron subordinados a la discusión de volúmenes de facturación.

La constitución de la Organización Mundial de la Salud (OMS) define salud como 'estado de complete bienestar físico, mental y social y no solo la ausencia de 
enfermedad o discapacidad'. Considerando la relación estrecha entre salud y entorno, Sterky et al. (1980) propusieron definir salud como "la condición humana, determinado por el ambiente social, económico, político y cultural". No cabía duda de la relevancia de esta definición al concluirse el conflicto armado con duración de 12 anos en Centro América, que costó la vida a por lo menos 200.000 personas y produjo un éxodo de más de un millón de refugiados desde El Salvador.

La "Declaración de Alma Ata” (1978) reconoció claramente la relación entre salud, entorno y desarrollo, apoyó el objetivo de Salud para Todos y concibió la APS como el mecanismo para alcanzar eso objetivo. Salud para Todos, en esencia, fue un llamado por la justicia social, la reducción de las desigualdades socioeconómicas y en salud de la población; la salud concebida como un derecho humano fundamental, esencial para el bienestar de individuos y el desarrollo sostenible de comunidades.

Es claro que existe una variedad confusa de perspectivas sobre lo que APS significa e implica en la práctica. Aunque la base filosófica de la APS fue definida en la "Declaración de Alma Ata", algunos consideran la APS únicamente como el nivel en el cual se presta la atención, el centro comunitario de salud o policlínica privada. Otros la conciben en términos de programas, como inmunizaciones, terapia de rehidratación oral, entre otros. Para otros, es una estrategia, pero es también una dirección o una filosofía. Como lo analiza también Mario Rovere, el problema es que la misma sigla 'APS' es usada (para comenzar en la "Declaración de Alma Ata" misma) para describir los diferentes aspectos:

- APS como filosofía (equidad social, desarrollo, autonomía);

- APS como estrategia (participación comunitaria, colaboración intersectorial y reorientación del sistema de salud);

- APS como nivel de atención (apropiado, efectivo o alcanzable); y

- APS como programa (limitado a ocho actividades esenciales).
La falta de una visión común y compartida del significado y propósito de la APS constituyó/e un gran reto. Se ha sugerido que una solución podría ser insistir en que el uso del concepto APS siempre debería ser seguido por una clarificación del mismo: nivel, programa, estrategia o filosofía. En el estudio "Rescate", sin embargo, se decidió no hablar de antemano de APS. Nicaragua había sido premiada en 1983 por la OMS y Organización Pan-american de Salud (OPS) por sus esfuerzos en desarrollar la APS mediante su sistema nacional universal de salud - creado a tres meses del triunfo de la revolución sandinista. El cambio de régimen era reciente, y se optó hablar de las 'experiencias locales innovadoras en salud' (ELIs). Sin embargo, es necesario aclarar (rescatar) "todos los pilares esenciales" de la APS.

En múltiples talleres con investigadores, profesionales de salud y tomadores de decisión en Nicaragua y en El Salvador, se profundizo la comprensión de conceptos como 'participación' (quiénes, cómo, en qué niveles, mecanismos, espacios), 'equidad', 'intersectorialidad', pero también 'calidad', 'relevancia' y 'sostenibilidad'. El caso de Guargila en El Salvador descrito en esta revista fue uno de aquellos identificados y seleccionados para el estudio a profundidad por el equipo de investigadores. Aunque el estudio no profundizó en aspectos financieros, este caso incluye la organización de un mecanismo de protección social popular.

El interés vigente aun después de tantos años en la APS se quedó claro al generarse la convocatoria para presentar expresiones de interés en la investigación por Teasdale Corti. América Latina fue la región que presentó mas propuestas; de un total de 41 se obtuvo (co)financiamiento para cinco proyectos de investigación, que están descritos en esta revista. El proceso de selección tomó en cuenta varios criterios, incluso la distribución geográfica, pero los estudios aprobados no pueden ser considerados representativos del conjunto.

Indudablemente, los sistemas de salud profundizan las inequidades y por lo tanto la coordinación vertical o la intrasectorialidad demanda atención igual como la coordinación horizontal o la intersectorialidad. Coincido con Mario Rovere que el momento actual demanda de una reorientación profunda en la educación 
de profesionales de la salud, en el abordaje de los determinantes sociales, la determinación social de la inequidad en salud.

La forma de mirar a la salud cambia y se vuelve más incluyente, y mientras que la Comisión de Determinantes Sociales evidenció con datos cuantitativos que para mejorar la salud y, en particular, la equidad en salud de la población es necesario ir más allá del sistema de prestación de servicios médicos, también el concepto del derecho a la salud se ha ampliado. En 2000, se actualizó el artículo 12, en el "Convenio de Derechos Económicos, Sociales y Culturales", reconociendo el derecho a la salud como incluyente, lo cual implica asegurar también las precondiciones esenciales, como ciertos servicios, bienes y condiciones para alcanzar el nivel más alto de salud. Se refiera a la atención de salud - acceso, atención apropiada - pero también al acceso al agua potable, saneamiento, nutrición, condiciones de empleo y de ambientes saludables, acceso a la educación y a la información. La obligación del estado es de proteger a la población contra la violación de estos derechos.

Las limitaciones de alcanzar el derecho a la salud están relacionadas con el compromiso político del gobierno; la brecha entre el discurso y la práctica por ejemplo por falta de recursos; la debilidad del sistema internacional de derechos humanos y la de los sistemas de salud, después de décadas de negligencia; la falta de inversión; los programas de ajuste estructural; la deuda y dependencia de lo cual limitan el desarrollo. La existencia de un sistema de salud fuerte y efectivo cada vez se reconoce más como precondición fundamental para alcanzar una sociedad saludable. Los sistemas y políticas de salud son un tema central en la agenda de muchos países y sociedades. Avances logrados en siglos pasados muchas veces a través de luchas sociales, en el desarrollo de sistemas de protección social y sistemas públicos de salud en países desarrollados, están amenazados. Es imprescindible comprender actores, procesos de tomada de decisión, incidir en las asimetrías de poder en un contexto de nuevos retos a nivel mundial. La necesidad de fortalecer la investigación de sistemas y políticas de salud y, en particular, la capacidad de la sociedad civil de conducir la investigación ya han sido ampliamente reconocidos. Un informe reciente de las Naciones Unidas (UNEP, 2012), que analiza los retos significando el cambio climático para la humanidad, identificó como problema crítico y como prioridad la necesidad de restablecer 'los puentes rotos entre la ciencia y la política'.

El proceso de investigación que hemos concluido en el marco del proyecto "Teasdale Corti" con apoyo del International Development Research Centre (IDRC) facilitó un intercambio y un proceso de aprendizaje no solo entre investigadores jóvenes, mentores, tomadores de decisión a nivel nacional, pero también entre equipos y representantes de movimientos sociales a nivel de América Latina e incluso de otros continentes. Sin duda, el material presentado en esta revista contribuirá al debate, a la reflexión y a la creación de nuevas alianzas.

\section{Referências}

DECLARAÇÃO DE ALMA-ATA: saúde para todos no ano 2000. 1978. Disponível em: <http://www.saudepublica.web.pt/05promocaosaude/Dec_Alma-Ata.htm>. Acesso em: 23 set. 2012.

MAHLER, $H$. The meaning of Health for All by the year 2000. World Health Forum, v. 2, n. 1, 1981, p. 05-22.

PEARCE, J. Violence, Power and Participation: Building Citizenship in Contexts of Chronic Violence No. 274. Brighton: Institute of Development Studies, 2007.
PROYECTO DE INVESTIGACIÓN RESCATE. Desarrollo alcanzado por los sistemas de salud en Guatemala, El Salvador y Nicaragua, centrado en los esfuerzos de la sociedad civil (1997-2000). Nicaragua: CIES; Holanda: KUN; EI Salvador: UES; Guatemala: INS; Bélgica: ITM, 2001. Informe Final.

RODRIGUEZ, C.; GARZÓN, G. La Atención Primaria en Salud en un modelo de prestación de servicios de salud orientado al mercado. In: BARTEN, F. et al. (Ed). Salud para Todos, una Meta Posible. Pueblos Movilizados y Gobiernos Comprometidos en un Nuevo Contexto 
Global. Construyendo prioridades para la investigación en APS con la sociedad civil. Buenos Aires: Informe ALCUEH, 2009. p. 149161.

STERKY, G. et al. Towards another development in health. In: RIFKIN, S.B. (Ed). Health: the human factor. Readings in health, development and community participation. Geneva: Christian Medical Commission: World Council of Churches, 1980. v. 3 (Contact Special Series, 3).
STUCKLER, D.; BASU, S.; MCKEE, M. Global health philanthropy and institutional relationships: how should conflicts of interest be addressed? PLoS Medicine, v. 8, n. 4, e1001020, 2011. doi:10.1371/journal.pmed.1001020.

UNITED NATIONS ENVIRONMENT PROGRAMME (UNEP). 21 Issues for the 21st Century: Results of the UNEP Foresight Process on Emerging Environmental Issues. Nairobi, Kenya: UNEP, 2012, 56p. 